Mots. Les langages du politique

Discours d'Amérique latine. Identités et conflits

\title{
Discours et stigmatisation identitaire
}

Le cas d'une communauté nahua au Mexique

Discourse and spoiled identity. The case of a Nahua community in Mexico

Discurso y estigmas identitarios. El caso de una comunidad nahua en México

Karla Janiré Avilés González

\section{OpenEdition}

\section{Journals}

Édition électronique

URL : https://journals.openedition.org/mots/22077

DOI : $10.4000 /$ mots. 22077

ISSN : 1960-6001

Éditeur

ENS Éditions

Édition imprimée

Date de publication : 30 novembre 2015

Pagination : 21-35

ISBN : 978-2-84788-776-1

ISSN : 0243-6450

Référence électronique

Karla Janiré Avilés González, " Discours et stigmatisation identitaire », Mots. Les langages du politique [En ligne], 109 | 2015, mis en ligne le 30 novembre 2017, consulté le 24 avril 2022. URL : http:// journals.openedition.org/mots/22077 ; DOI : https://doi.org/10.4000/mots.22077 


\section{Discours et stigmatisation identitaire. Le cas d'une communauté nahua au Mexique}

En Amérique Latine, comme sous d'autres latitudes, les locuteurs des langues minorisées sont souvent exposés à une stigmatisation qui entraîne la reconfiguration de leurs identités et de leurs pratiques sociolinguistiques, y compris la disparition de la langue en question (Avilés González, 2011). Kenneth Hale a dénoncé le fait que les langues et les cultures politiquement dominantes écrasent les langues et les cultures indigènes locales (Hale, 1992, p.1). Les sociolinguistes catalans des années soixante-dix ont eux aussi décrit cette situation (Ninyoles, 1997 [1971]) et plusieurs travaux récents sur les langues et les cultures menacées d'extinction l'ont eux aussi analysée (Léonard, Avilés González, 2015). Il s'agit d'un conflit glottopolitique, c'est-à-dire d'une « action [conflictuelle] de la société sur le langage» (Guespin, 1984, p.13). Ce phénomène se révèle la plupart du temps traumatique pour les locuteurs stigmatisés et n'est pas silencieux : il s'exprime dans des manifestations d'ordre linguistique ou paralinguistique qui, même dans certains cas de non-verbalisation, ont du sens (Barbet, Honoré, 2013). Le cas de la communauté de Santa Catarina (Morelos, Mexique), où la langue originaire - le nahuatl - est au bord de l'extinction, nous montre combien sont importantes les réactions discursives aux stigmates : elles témoignent du rejet d'une identité dite indigène imposée de manière externe par le groupe hégémonique, mais aussi du rejet, par ses locuteurs eux-mêmes, de la langue minorisée (Avilés González, 2011)ํ․ Autointerruptions, silences pesants, formules fatalistes, stratégies rhétoriques, évitement... Les récits de vie trahissent des regrets, une amertume et parfois de la colère, face à la rupture du veto catégorique auxquels ils sont soumis. Ce veto est défini par Lluís Aracil (1983, p. 108) comme «un refoulement insidieux du conflit [glottopolitique], qui interdit d'en faire un objet de réflexion et de débat»; il «empêche de construire un épilangage de la situation » et par la suite de transcender le conflit. L'analyse de ce genre de stigmates nous invite

1. En accord avec les locuteurs contactés, on évite l'emploi du terme indigène pour parler de l'identité sociale de cette communauté. Au-delà des débats sur la pertinence de ce concept, il n'est pas question, dans ce travail, d'imposer des définitions que les communautés concernées réfutent.

Labex EFL, Université Sorbonne Paris Cité, Paris 7 karla.j.aviles@gmail.com 
à réfléchir sur les expressions discursives révélatrices de ces conflits. Elle permet aussi d'analyser les façons par lesquelles le chercheur, en tant que participant aux interactions, peut contribuer à leur émergence, ainsi qu'à un éventuel processus de catharsis identitaire pour les locuteurs - par exemple, lors de la réalisation d'entretiens semi-structurés au cours desquels les stigmates sont thématisés. Cet article remet ainsi en question l'objectivité du positivisme canonique. Au-delà d'une description d'interactions discursives marquées par la mémoire de la stigmatisation, il s’interroge sur la place du chercheur et sur son impact : la subjectivité n'est pas perçue, ici, par opposition à un objet d'étude indépendant de l'observateur, mais par rapport à une relation entre acteurs sociaux, entre êtres humains, qui inclut le chercheur².

\section{Identités sociales, identités linguistiques}

Àl'heure des revendications ethniques contemporaines, caractérisées par l'affirmation que les populations font de leurs distinctions ethniques, les études centrées sur l'identité des sociétés amérindiennes sont en plein essor. Elles se focalisent souvent sur les discours politiques produits dans l'espace public et dans une situation sociale formelle (voir entre autres Sierra, 2012)3. Un exemple topique en est la médiatisation que l'Armée zapatiste de libération nationale 4 a réalisée, depuis 1994, des droits à l'autonomie des peuples indigènes mexicains : elle a contribué à la reconnaissance constitutionnelle, en 2001, de la diversité linguistique et culturelle de la nation, et à la création en 2003 de la loi des droits linguistiques des peuples indigènes. Malgré ces avancées constitutionnelles, les questions d'ordre glottopolitique sont toujours d'actualité au Mexique, pays marqué par une longue histoire de minorisation des langues et des cultures originaires (Barriga, Butrageño, 2010). Ces dernières sont considérées, aujourd'hui, comme des langues menacées d'extinction face au bilinguisme substitutif en cours et alors que l'espagnol reste, à ce jour, la seule langue officielle (Avilés González, 2011).

Dans le cas présenté ici - le nahuatl de Santa Catarina -, les discours politiques concernant la préservation de la langue menacée ont été médiatisés récemment (Camacho, 2015). Ils font partie d'une glottopolitique officielle centrée sur l'enseignement formel du nahuatl dans la région, qui n'établit pas de planification sociolinguistique adaptée aux besoins de chaque communauté.

2. Ces questions d'ordre épistémologique et méthodologique ont été développées dans plusieurs travaux, dont Léonard et Avilés González (2015) et Avilés González (2015).

3. On considère qu'une situation sociale est formelle par opposition aux situations plus intimes et spontanées (Irving, 2009).

4. Acronyme EZLN en espagnol. Cette médiatisation concerne aussi bien les journaux que la radio et la télévision, au format analogique et numérique. 
Ils n'évoquent pas la question des matériaux pédagogiques ou les moyens d'assurer la transmission linguistique intergénérationnelle - indispensable pour mettre un frein à l'extinction des langues minorisées (Fishman, 1991). Ces discours sont un bon exemple du «problème de l'idéalisation » dénoncé par la sociolinguistique catalano-occitane : ce sont des «idéologies compensatoires » qui donnent l'impression qu'une action est entreprise en faveur des peuples minorisés sans pour autant remettre en cause le pouvoir de la société dominante. Ils perpétuent ainsi le conflit diglossique (Ninyoles, 1997, p. 90) qui oppose la société métisse hispanophone et les locuteurs du nahuatl.

Une mission de recherche réalisée en 2015, au Mexique, m'a permis de constater qu'aucune action officielle n'est mise en place à Santa Catarina pour revitaliser le nahuatl, très menacé dans cette communauté. Les nahuatophones actifs représentent environ vingt pour cent du total de la population. Ce sont surtout des adultes âgés de soixante ans et plus, des paysans nommés comuneros, propriétaires de leurs terres par droit historique. Les enfants sont hispanophones monolingues, à l'exception de quelques cas particuliers. Les fonctions du nahuatl sont restreintes aux contextes informels (par exemple la vie familiale). II n'y a pas d'activation de la langue durant l'adolescence ou lors d'autres périodes de la vie adulte. Tout montre donc que cette langue requiert une attention immédiate. Divers facteurs ont fait que les initiatives communautaires qui visaient à préserver la langue originaire n'ont pas réussi à assurer la transmission linguistique intergénérationnelle. Parmi eux, les stigmates envers le nahuatl et ses locuteurs jouent un rôle important (Avilés González, Flores Farfán, 2010).

Peu d'études ont été réalisées sur les discours glottopolitiques produits dans la sphère informelle des peuples amérindiens : dans les foyers, dans les interactions spontanées, dans la vie quotidienne (Hill \& Hill, 1999). Cette dernière est pourtant considérée comme l'espace communautaire par excellence, celui de la reproduction et de l'innovation sociale, où émerge une réflexivité citoyenne contestataire qui érode le pouvoir dominant, où la transformation sociale se concrétise (Reguillo, 2000). Il est donc pertinent de repérer comment les locuteurs réagissent dans le discours quotidien aux idéologies ethno-linguicides mises en place dans la région. Ce travail montrera que les retombées de ces discours ne sont pas négligeables. Ils font écho à une stigmatisation identitaire de longue durée, avec un effet important sur les reconfigurations des pratiques sociolinguistiques (Avilés González, 2011).

\section{Approche méthodologique}

Les interactions avec les locuteurs de Santa Catarina le montrent : parler de la stigmatisation de leur langue maternelle touche des fibres affectives très 
sensibles. Ces réactions se manifestent dans toutes les sphères du langage, y compris dans les silences. Celui-ci est en effet «[un] élément constitutif de la communication », [un] mécanisme producteur de sens » (Barbet, Honoré, 2013, p. 11). Les expressions populaires le disent bien : le silence peut être «parlant» même s'il s'agit d'un «silence de mort». Ceux que nous analyserons ici sont surtout d'ordre pragmatique (voir plus loin ; Ephratt, 2011). Nous nous concentrerons sur la construction de la phrase, le contenu du discours, les formules thématiques et la prosodie. L'échantillon examiné (trois fragments de trois histoires de vie) provient d'une étude réalisée entre 2003 et 2005 sur la stigmatisation du nahuatl à Santa Catarina (Morelos, Mexique). Il est extrait d'un corpus rassemblant quinze histoires de vie des membres d'une même famille et de leurs amis, lesquelles représentent l'expérience que trois générations ont faite des stigmates sociolinguistiques. Les dialogues privilégiés pour cette analyse correspondent aux réactions les plus fréquentes que l'on peut détecter dans leurs narrations. J'ai recueilli ces histoires de vie de janvier à avril 2005 , à travers des conversations relativement spontanées, avec l'autorisation ex post facto des locuteurs. Elles sont survenues lors de confidences entre les locuteurs du nahuatl et l'étudiante que j'étais à l'époque, mais aussi lors de "visites " ${ }^{5}$. Lorsque je rencontrais de nouveaux locuteurs, ils me posaient souvent des questions sur mon identité, en tant qu'étrangère à la communauté. Il s'agit de ce que l'on pourrait appeler une épreuve, que les locuteurs mettent en place afin de négocier des identités en conflit - en prenant en compte, dans mon cas, le fait que j'étais une étudiante hispanophone intéressée par l'apprentissage d'une langue minorisée au niveau local, régional et national (Avilés González, 2015). La présentation de mon identité n'était pas insignifiante : mon intérêt pour la compréhension du «comment on vit » et «comment on pense le nahuatl ${ }^{6}$ devenait souvent l'instrument d'une réflexion autour des stigmas sociolinguistiques. J'ai analysé les réactions discursives d'après une approche ethnographique de la communication (Hymes, 1974), c'est-à-dire comme le produit mais aussi comme la conséquence d'une séquence particulière d'actes communicatifs, marqués entre autres par l'identité des participants et les contextes d'interaction. Ces réactions font partie de normes, ou plutôt de schèmes communicatifs relativement stables, quoique flexibles (Briggs, 1990). Ces schèmes, à leur tour, procèdent de cadres d'interaction spécifiques, socio-culturellement différenciés (Goffman, 1991), qui reproduisent et transforment la réalité sociolinguistique, y compris la réalité idéologique. Le présent travail met ainsi en évidence l'existence d’idéologies et de préjugés linguistiques qui ne sont pas objectifs ou naturels, mais qui sont naturalisés

5. Les visitas sont réalisées au foyer, par les amis, familiers et connaissances, afin d'entretenir les liens sociaux tels que les relations de réciprocité.

6. «Cómo se vive y piensa el náhuatl de Santa Catarina ». Sauf mention contraire, toutes les traductions sont de l'auteure. 
(Silverstein, 1996). Ces derniers, construits sur la base du langage, contribuent au développement d'une identité stigmatisée et fragmentée. Les comportements discursifs des locuteurs apparaissent ainsi comme des réactions aux conflits glottopoliques. Tout en formulant les axiomes qui rendent une certaine congruence idéologique à la stigmatisation, elles écrasent et étouffent la langue minorisée. Cependant, on sait que le fait de parler d'un trauma fait partie d'un processus thérapeutique. Puisque la stigmatisation engendre des traumas, dans quelle mesure en parler est-il un moyen de faire émerger une catharsis identitaire qui la conteste?

\section{Du mutisme à la catharsis}

Les réactions étudiées montrent en particulier que le silence a une forte valeur communicative, surtout pragmatique (Ephratt, 2011), car il est lié aux sujets qu'on ne doit pas évoquer, comme c'est le cas avec les identités stigmatisées. Certaines formes du silence font partie de ce qu'Erving Goffman (2001[1963]) appelle les stratégies d'ajustement, entendues comme des actes et attitudes que les acteurs stigmatisés - ou ceux qui sont susceptibles de l'être - développent afin d'assumer les comportements que les acteurs réputés normaux attendent d'eux'. Dans le cas étudié ici, l'emploi de la langue minorisée devient le principal signe à masquer : la mort linguistique étant une forme de silence perpétuel, cette attitude est sans doute la plus évidente et la plus radicale des réactions développées par les locuteurs afin d’éviter la stigmatisation sociolinguistique.

Nous avons constaté cette réaction à Santa Catarina, mais d'autres formes de silence, moins évidentes bien que puissantes, apparaissent aussi comme des stratégies d'ajustement. C'est le cas des longues pauses qui se terminent en silence fermé, ou de celles qui indiquent un silence réflexif, une autocorrection, une autocensure, une hésitation, une interruption et qui modifient à divers degrés la forme et le contenu du discours (par exemple par changements de topique). Elles sont l'expression d'un blocage communicatif, lui-même conséquence d'une interdiction psycho-sociale souvent implicite, décrite plus haut sous le nom de veto catégorique. Ce veto empêche la construction d'un discours secondaire, réflexif, qui viserait à dépasser la stigmatisation. De cette façon, ces silences entretiennent une structure asymétrique de pouvoir sociolinguistique (Ninyoles, 1997). L'interaction suivante le montre clairement; deux

7. La distinction entre acteurs normaux ou stigmatisés n'est pas dichotomique, ni discrète, mais heuristique. Les acteurs stigmatisés, dans certains contextes, peuvent jouer le rôle d'acteurs normaux tout en stigmatisant d'autres locuteurs (et vice-versa). C'est le cas des locuteurs bilingues nahuatl-espagnol qui, tout en parlant une variante de l'espagnol fortement influencée par le nahuatl, stigmatisent les nahuatophones plus actifs. 
femmes bilingues qui parlent couramment nahuatl (A : 66 ans; $B$ : 50 ans) et moi-même (I: 26 ans à l'époque) discutons de l'absence d'intérêt des nouvelles générations pour apprendre le nahuatl et des conflits sociolinguistiques qu'elles ont vécus ${ }^{8}$ :

\begin{tabular}{|c|c|}
\hline Version originale (Vo) & Version française (VF) ${ }^{9}$ \\
\hline A 1 : aprender en., del náhuatl.. & A 1: apprendre en., du nahuatl.. \\
\hline I 2 :a-ha & I 2 : oui \\
\hline $\begin{array}{l}\text { A } 3: \text { y como ahora otros ya no, ya no } \\
\text { procuran pues, ya no// }\end{array}$ & $\begin{array}{l}\text { A } 3 \text { : et comme aujourd'hui les autres [gens] } \\
\text { ne cherchent plus, non, ne cherchent plus, } \\
\text { quoi [à l'apprendre]// }\end{array}$ \\
\hline B 4 : se les olvida, ya no pues... & $\begin{array}{l}\text { B } 4 \text { : ils l'oublient [les enfants, d'autres } \\
\text { gens oublient le nahuatl], [ils ne le parlent] } \\
\text { plus quoi... }\end{array}$ \\
\hline $\begin{array}{l}\text { A } 5 \text { : ya no hacen caso.. ya no.. ya no se } \\
\text { acuerdan, van a., ya no pueden hablar así.. } \\
\ldots\end{array}$ & $\begin{array}{l}\text { A } 5 \text { : ils ne font plus attention.. non... non.. } \\
\text { ils ne se rappellent plus, ils vont a., ils ne } \\
\text { peuvent plus parler ainsi [nahuatl]..... }\end{array}$ \\
\hline $\begin{array}{l}\text { I } 6: \text { mmhm... pues sí yo creo que si todos } \\
\text { nos organizamos acá y empezamos a } \\
\text { hablar otra vez náhuatl hay mucha gente.. } \\
\text { interesada a venir a aprender. }\end{array}$ & $\begin{array}{l}\text { I } 6: \text { mmhm... bah oui je crois que si nous } \\
\text { tous nous organisons ici et commençons } \\
\text { à parler à nouveau en nahuatl, il y a } \\
\text { beaucoup de monde.. intéressé à venir à } \\
\text { l'apprendre. }\end{array}$ \\
\hline $\begin{array}{l}\text { B } 7: \text { bueno los de afuera pero luego va uno } \\
\text { a alguna parte y luego nada más se ríen de } \\
\text { uno... }\end{array}$ & $\begin{array}{l}\text { B } 7: \text { bon les étrangers mais ensuite on va } \\
\text { quelque part et puis [les gens] se moquent } \\
\text { de nous... [quand on parle nahuatl], (ton de } \\
\text { chagrin). }\end{array}$ \\
\hline I $8:$ ¿sí? & I8: oui? \\
\hline A 9: sí & A 9: oui \\
\hline I 10 : por ejemplo, ¿cuándo le ha pasado? & $\begin{array}{l}\text { I } 10 \text { : par exemple, quand est-ce que ça } \\
\text { vous est arrivé? }\end{array}$ \\
\hline $\begin{array}{l}\text { B } 11 \text { : (cambio de tópico) en alguna parte } \\
\text { ya casi casi no lo ocupan... los de Tepoztlán } \\
\text { ya no, y acá a vec[es].. las abuelitas si a } \\
\text { uno le hablan cuando así en náhuatl, uno } \\
\text { les contesta. i¿Pero los que no saben?! ¡Ay! } \\
\text { Luego se empiezan a reír: - ¿ay qué cosa }\end{array}$ & $\begin{array}{l}\text { B } 11 \text { : (changement de topique) quelque } \\
\text { part il n'est quasiment plus, quasiment } \\
\text { plus employé... Ceux de Tepoztlán [ne } \\
\text { l'utilisent] pas plus, et ici parf[ois].. les } \\
\text { quelqu'un en nahuatl, on leur répond. Mais } \\
\text { ceux qui ne savent pas [parler nahuatl]?! }\end{array}$ \\
\hline
\end{tabular}

8. La notation employée est la suivante : In : Incompréhensible; [ ] : Mots manquants; () : Précisions sur les contextes, référents et formes du discours ; // : Interruption ; - : Répétition d'une lettre, mot ou phrase; , : Pause brève ; .. : Pause moyenne ; ., : Pause brève suivie d'un changement dans la forme ou le contenu du discours ; ... : Pause longue ou prolongée.

9. Afin de maintenir une traduction cohérente vis-à-vis de l'analyse linguistique ici réalisée, nous essayons de respecter dans la version française la variété de l'espagnol employée par les locuteurs. Laquelle, comme cela a été précisé ci-dessus, s'éloigne parfois des règles de l'espagnol standard. Il y aura donc aussi des éloignements morphosyntaxiques par rapport au français standard.

10. Les dilemmes de traduction associés avec la forme uno de l'espagnol au français sont assez importants en termes analytiques. Ils seront donc abordés plus loin dans le corps du texte. 
dicen? ¡Ni sé!... [se dicen los que no saben]-. Pues la verdad pues, no saben.
Aïe! Ensuite ils commencent à rire: Aïe qu'est-ce qu'elles racontent? Je n'en sais rien !... [se disent ceux qui ne savent pas]-. Effectivement, ils ne savent pas parler [nahuatl] quoi.

I 12 : de eso se trata [de aprender náhuatl] y en cambio la gente que se ríe pues no sabe nada y...

12 : il s'agit de ça [d'apprendre le nahuatl] et en retour les gens qui se moquent, et bien ils ne savent rien quoi et...

A 13 : no sabe nada. Na'mas por que se ríe, se pone a reír puede que diga: -¡Ay, qué están diciendo!-... Es que no más uno lo ve, uno habla así como los de Cuentepecc. Ansí hablan ¿verdad?

A 13 : ils ne savent rien. Seulement parce les gens rigolent, ils se mettent à rigoler, et il arrive qu'ils disent [les hispanophones] : «Aïe, qu'est-ce qu'ils sont en train de dire! [les hispanophones] »... C'est qu'il n'y a que nous qui les voyons [les stigmas], on parle ainsi comme ceux de Cuentepec. Ils parlent ainsi [nahuatl], n'est-ce pas?

B 14 : a-ha. B 14 : oui.

A 15 : y a., en Cuernavaca na' más se miran. También se burlan de uno... (tono de lamento y molestia).

I 16 : ¿sí? ¿Qué le dicen?

A 17 : pues así (In) quién sabe.. qué cosa dicen. Sí pues creo que se ve. Hay que disimular, aunque no es así.. tienes que hablar de., al salir de aquí tienes que hablar en- en- en español porque si no no.
A 15 : et en., à Cuernavaca ils ne font que se regarder. Eux aussi ils se moquent de nous... (ton de tristesse et d'agacement).

I 16 : oui ? Qu'est-ce qu'ils vous disent?

A 17 : ainsi quoi (In) je n'en sais rien.. quel truc ils racontent? Oui, quoi, je crois que ça se voit. Il faut dissimuler, bien que ce n'est pas comme ça [ça ne devrait pas être comme ça].. Tu dois parler de., quand tu sors d'ici tu dois parler en- en- en espagnol parce qu'autrement non, non.

Le thème de cet échange est d'ordre linguistique, et le code employé est aussi le code dominant (le castillan). Les locutrices natives, face à une participante extérieure qui utilise la variété de l'espagnol la plus prestigieuse du Mexique (celle de Mexico), développent donc une extrême conscience de leur propre façon de parler espagnol - une variété linguistique stigmatisée en raison d'une influence nahua. Ces phénomènes s'expriment, de manière inconsciente, dès le premier tour de parole, à travers la substitution du morphème del («du») à en ("en »), laquelle est marquée par une pause suivie de cette reconstruction syntaxique (A 1$)$. Ce qui produit un sens différent de l'intention communicative de la locutrice, à savoir : aprender nahuatl («apprendre le nahuatl »), à propos de quoi ce dialogue s'était engagé.

Comme on le verra plus loin, l'autocorrection n'est pas la seule réaction aux stigmates. Diverses formes de silence sont précédées d'un autre signal discursif, révélateur d'un sentiment fataliste : la répétition insistante de l'adverbe de temps négatif ya no «(ne) .... plus». Celui-ci, qui met en évidence, sur le plan aspectuel, la rupture d'une continuité temporelle, évoque l'extinction du nahuatl («les gens ne cherchent plus à apprendre la langue originaire », cf. A 3) (Alarcos, 2002, p.165). Telle qu'elle est employée dans cet exemple, 
l'expression ya no devient partie d'une formule ${ }^{11}$ fataliste, utilisée afin d'accorder une certaine congruence à cette disparition progressive - sans pour autant thématiser les conflits linguistiques. L'interruption en B 4 confirme le propos. À ce stade, les locuteurs ne se demandent ni pourquoi la population ne cherche plus à parler sa langue, ni pourquoi le nahuatl ne se transmet pas aux nouvelles générations. Ainsi, les locuteurs de langues minorisées construisent une «théorie de la congruence» (Muñoz, 1987), un discours idéologisé qui, malgré leur haut niveau de conscience linguistique, bloque les réflexions épilinguistiques sur les langues en compétition. Il s'agit d'une rationalisation, stratégie destinée à éviter le conflit produit par le veto catégorique. Cet échange montre que le chercheur, tout en participant aux interactions, joue un rôle important dans la rupture de ce veto en déclenchant parfois des processus cathartiques. Cependant, il peut aussi provoquer des interruptions qui contrarient les silences réflexifs. Le premier cas est flagrant dans la séquence I 6, où la participante (moi-même) expose une alternative, naïve et inattendue, à l'extinction du nahuatl : faire en sorte que la communauté se remette à parler cette langue en raison de l'intérêt que lui portent les étrangers. Cette naïveté, plutôt un signe de l'intérêt du chercheur pour revitaliser la langue minorisée, a déclenché le dévoilement spontané du sujet tabou : le conflit sociolinguistique.

Ainsi, dans la réplique suivante, $B 7$ décrit, avec des prosodèmes marquant la tristesse et sans pauses d’hésitation, le mépris enduré par les locuteurs natifs face aux habitants des communautés plus prestigieuses comme Cuernavaca et Tepoztlán (centres politiques plus importants de la région; voir B 11, A 15). Ce mépris donne lieu à des sarcasmes et à tout un «langage de la moquerie » (Hill, 2001; Avilés González, 2011) auquel sont exposés les habitants de Santa Catarina. Au cours de l'interaction, le conflit sociolinguistique est ainsi plusieurs fois dénoncé (cf. B 11, A 13, A 15), mais les deux locutrices utilisent une expression particulière pour thématiser ces tensions : le pronom indéterminé uno, dont la référence est variable et parfois ambiguë. Uno peut se substituer en effet à la première personne du singulier (moi) et à la première personne du pluriel (nous); sur le plan référentiel, il est comparable au pronom indéfini (quelqu'un) et au sujet indéfini de la troisième personne du singulier (on) du français. Cette ambigüité référentielle constitue l'un des traits les plus riches du discours sur la stigmatisation, car les locutrices parlent des conflits auxquels elles sont confrontées sans faire d'allusion directe à elles-mêmes. Par cette distance, elles parviennent à rompre le veto catégorique, tout en thématisant les stigmates.

Si l'on prend en compte l'intensité affective de ces conflits identitaires, de tels blocages communicatifs sont une réaction tout à fait compréhensible. Ce

11. La «formule» est ici définie comme l'ensemble des expressions qui articulent le sujet thématisé avec la forme du discours au niveau morphosyntaxique, prosodique et rhéthorique. C'est une manière de figer le sens par la voie de la répétition (Franco, 2004, p. 90-91). 
qui est inattendu et parfois imprévisible, c'est la forme par laquelle ils vont se manifester dans les énoncés. Dans le cas examiné, c'est l'absence de réponse à la question, posée par I 10, "Quand est-ce que ça vous est arrivé? ». Face à cette question par laquelle l'observatrice l'invite à sortir de l'indéfinition et à communiquer un exemple personnel, la locutrice $B 11$ réagit par un changement de sujet suivi d'une explication toujours indirecte, mais plus concise, de la stigmatisation vécue. Les noms des endroits où ces conflits ont été expérimentés apparaissent alors entre pauses réflexives, mots coupés, formes indéfinies, associés à des phrases exclamatives et à des interjections qui témoignent de l'agacement des locutrices confrontées à la stigmatisation, et de leur contestation de celle-ci. Nous comprenons alors que les stigmates procèdent principalement d'une absence de savoir des acteurs normaux, d'un déficit de compétence linguistique nahua mais aussi d'une carence de compréhension interculturelle. La locutrice dénaturalise ainsi les idéologies linguistiques autour du nahuatl : elle découvre le caractère fallacieux de la subordination de cette langue minorisée, tout en mettant en évidence les causes de l'extinction linguistique. En résumé, les représentations épilinguistiques soutiennent une violence symbolique (Bourdieu, 2001) avec des conséquences importantes sur l'identité collective et sur la langue originaire de Santa Catarina. La souffrance identitaire s'affirme comme la cause la plus évidente du remplacement du nahuatl par l'espagnol.

Les marques discursives identifiées dans cette interaction reviennent, de manière plus ou moins systématique, dans les quinze récits de vie recueillis à Santa Catarina. Elles ne sont pas propres à la génération des bilingues âgés de plus de 50 ans. On les retrouve aussi dans l'histoire de vie d'un locuteurâgé de 37 ans, qui discute avec moi de la pratique de cette langue dans sa génération :

\begin{tabular}{|c|c|}
\hline vo & VF \\
\hline $\begin{array}{l}\text { C } 19 \text { : la gente con la que uno trata ya... } \\
\text { ese dialecto, ya no.. lo conocen, o ya no } \\
\text { lo hablan. Por decir, si usted.. sale.. fuera } \\
\text { a trabajar por decir vamos a suponer a la } \\
\text { ciudad de México, pues ahí la gente no } \\
\text { habla náhuatl ya... entonces la gente se } \\
\text { tiene que adaptar a la forma.. de cómo vive } \\
\text { la- la demás gente, ¿verdad? Mire, la verdad } \\
\text { la verdad, en esa generación que crecimos } \\
\text { nosotros ya nadie hablaba náhuatl }\end{array}$ & $\begin{array}{l}\text { C } 19 \text { : les gens avec qui on est en contact... } \\
\text { ce dialecte-là [le nahuatl], ils ne.. ils ne le } \\
\text { connaissent plus, ou ils ne le parlent plus. } \\
\text { On va dire, si vous.. sortez... travailler } \\
\text { dehors [en dehors de Santa Catarina], on } \\
\text { va dire à Mexico, eh bien là-bas les gens } \\
\text { ne parlent plus nahuatl... alors les gens } \\
\text { doivent s'adapter à la façon.. de vivre des - }- \\
\text { des autres gens, n'est-ce pas? Regardez, à } \\
\text { vrai dire, à vrai dire, dans notre génération } \\
\text { plus personne ne parlait nahuatl }\end{array}$ \\
\hline I 20 : ¿ya nadie hablaba náhuatl? & I 20 : plus personne ne parlait nahuatl? \\
\hline C $21:$;nadie! & C 21: personne! \\
\hline | $22:$ i¿a poco?! & I $22:$ ah bon?! \\
\hline C $23:$ ¡nadie! & C 23: personne! \\
\hline
\end{tabular}




\begin{tabular}{|c|c|}
\hline I 24 : ¿los niños no hablab[an?]// & I 24 : les enfants ne le parla[ient pas?]// \\
\hline C 25 : jnadie! Nadie hablaba náhuatl.. sí & $\begin{array}{l}\text { C } 25 \text { : personne! Personne ne parlait } \\
\text { nahuatl... oui }\end{array}$ \\
\hline $\begin{array}{l}\text { I } 26 \text { : ¿y cuándo algún adulto llegaba y le } \\
\text { hablaba náhuatl a alguno de ustedes, qué } \\
\text { hacían ustedes? }\end{array}$ & $\begin{array}{l}\text { I } 26 \text { : et lorsque un adulte arrivait et parlait } \\
\text { nahuatl à l'un d'entre vous, qu'est-ce que } \\
\text { vous faisiez? }\end{array}$ \\
\hline $\begin{array}{l}\text { C } 27 \text { : pues mire.. pues por lo regular ya } \\
\text { no se habló en náhuatl a nosotros, sí.. o } \\
\text { como mi abuelo sabe hablar náhuatl y sabe } \\
\text { hablar bien el español, cuando nosotros } \\
\text { cre[cimos]., nos crecimos ya mi abuelo ya } \\
\text { no nos hablaba náhuatl.. }\end{array}$ & $\begin{array}{l}\text { C } 27 \text { : eh bien.. bon, en règle générale, on } \\
\text { ne nous a plus parlé nahuatl, à nous, oui.. } \\
\text { c'est comme mon grand-père qui sait parler } \\
\text { nahuatl et sait bien parler l'espagnol, } \\
\text { lorsque nous avons gra[ndit]., nous avons } \\
\text { grandi à ce moment-là mon grand-père ne } \\
\text { nous parlait plus nahuatl.. }\end{array}$ \\
\hline I $28:$ ¿ya no les hablaba? & I 28 : il ne vous parlait plus? [nahuatl] \\
\hline $\begin{array}{l}\text { C } 29 \text { : ya mire.., aunque no me crea la gente } \\
\text { que- que hablaba náhuatl como que la } \\
\text { hacían sentir menos.. [con respecto a los } \\
\text { hispanohablantes].. }\end{array}$ & $\begin{array}{l}\text { C } 29 \text { : eh bien.., croyez-moi ou non mais } \\
\text { les gens qui- qui parlaient nahuatl, on leur } \\
\text { faisait éprouver un sentiment d'infériorité.. } \\
\text { [par rapport aux hispanophones].. }\end{array}$ \\
\hline I 30 : ¿ah sí? & I30: ah bon? \\
\hline C $31:$ la verdad por// & C 31 : pour de vrai, car// \\
\hline I 32 : ¿qué le decían?// & I 32 : qu'est-ce qu’ils vous disaient?// \\
\hline $\begin{array}{l}\text { C } 33 \text { : eso se- se dejó esa costumbre., pos } \\
\text { ya, pos.. todavía.. todavía tenía una.. } \\
\text { ¿cómo le diré?.. ¿le explicaré? Todavía la } \\
\text { de[más]., la gente..., se decía que... que no } \\
\text { tenía.. que no tenía......... ¿ ¿cómo le diré?, } \\
\text { de cierta forma... o sea como que la hacían } \\
\text { sentir menos por hablar ese idioma.. o ese } \\
\text { dialecto }\end{array}$ & $\begin{array}{l}\text { C } 33 \text { : [c'est pour] ça qu'on a abandonné } \\
\text { cette coutume., bah déjà, bah... il y avait } \\
\text { encore.. encore..... comment dire?.. } \\
\text { comment expliquer? Les au[tres].. les } \\
\text { gens.., ils disaient que... qu'on n'avait } \\
\text { pas.. qu'on n'avait pas.......... comment } \\
\text { dire?, d'une certaine manière... c'est-à- } \\
\text { dire c'est comme si on leur faisait éprouver } \\
\text { un sentiment d'infériorité parce qu'ils } \\
\text { parlaient cette langue-là... ou ce dialecte-là }\end{array}$ \\
\hline
\end{tabular}

Ici, le conflit sociolinguistique est mis en évidence dès le début de l'interaction (C 19). Le locuteur affirme que la disparition du nahuatl est due aux conflits entre hispanophones et nahuatophones sur le marché de l'emploi, et par la suite aux conflits entre des formes de vie antagoniques. Le non-dit est alors constaté ethnographiquement : à Santa Catarina le bilinguisme nahuatlespagnol, ainsi que le monolinguisme nahuatl, ne sont pas envisagés comme un avantage pour accéder à un emploi en milieu urbain. L'extinction du nahuatl est donc inévitable afin que les communautés qui le parlent s'adaptent aux formes de vie des autres, les citadins. On remarque que le conflit sociolinguistique est ici signifié par les mêmes réactions discursives que celles que nous avons analysées plus haut. Dans cet échange, le locuteur natif emploie à cinq reprises l'expression ya no «(ne) ... plus» en rapport avec l'absence de l'apprentissage du nahuatl dans sa génération. Il recourt lui aussi à la forme indéterminée de l’espagnol uno (ici on) : «les gens avec qui on est en contact... ». 
II marque des pauses réflexives qui indiquent la présence des blocages communicatifs liés à la rupture des tabous relatifs à ce sujet : « ... ce dialecte-là [le nahuatl], ils ne.. ils ne le connaissent plus ».

La séquence communicative I 20-C 29, où le chercheur remet en question le fait que «plus personne ne parlait nahuatl » à Santa Catarina, exprime par rapport au vécu du locuteur un doute que celui-ci conteste par un argument d'autorité : «croyez-moi ou non mais les gens qui- qui parlaient nahuatl, on leur faisait éprouver un sentiment d'infériorité.. » (C 29). Il fait ainsi remarquer à la fois que certains locuteurs parlaient nahuatl à son époque, et le mépris enduré par ces locuteurs face aux hispanophones - donc les asymétries du pouvoir entre ces groupes sociolinguistiques. Il est clair, ici, que le conflit sociolinguistique est aussi un conflit entre identités collectives, matérialisé par des stigmates. Ce qui est confirmé par C 33, sous l'aspect de longues pauses et de mots coupés. Ces réactions discursives sont le préambule qui permet au locuteur de thématiser le sujet tabou - le fait que la langue originaire n'est plus parlée à cause de sa stigmatisation, dont il est un témoin. Dans cette interaction, les stigmates ne sont pas dépassés. L'extinction de la langue reste naturalisée, tout comme la violence symbolique à laquelle les locuteurs se sont accoutumés.

Ces phénomènes se retrouvent chez d'autres habitants de Santa Catarina, moyennant les nuances de chaque histoire de vie. Témoin l'interaction suivante, où une femme qui comprend le nahuatl mais ne le parle pas $(\mathrm{H}, 26$ ans), une femme bilingue ( $D, 55$ ans) et moi-même évoquons les inconvénients suscités par l'appartenance à un groupe stigmatisé :

\begin{tabular}{l|l}
\hline Vo & VF \\
\hline $\begin{array}{l}\text { I } 34: \text { y... ¿a ti alguna vez te han dicho india } \\
\text { o María o algo así? }\end{array}$ & $\begin{array}{l}\text { I } 34: \text { et... à toi ils t’ont déjà dit indienne ou } \\
\text { Maria ou quelque chose du genre? }\end{array}$ \\
\hline D $35:$ i¿verdad? de que., jen Cuernavaca sí! & D $35:$ c'est vrai ? que., à Cuernavaca oui ! \\
\hline H $36:$ nos dicen así.. & H $36:$ ils nous appellent comme ça.. \\
\hline I $37:$ ¿sí? & I $37:$ oui? \\
\hline $\begin{array}{l}\text { D } 38: \text { sí.. « ¿por qué hablas así india?» [les } \\
\text { ¿dicen los hispanohablantes] }\end{array}$ & $\begin{array}{l}\text { D } 38: \text { oui.. « pourquoi tu parles } \\
\text { comme ça, indienne?» [leur disent les } \\
\text { hispanophones] }\end{array}$ \\
\hline I $39:$ ¿y qué haces cuando te dicen eso? & $\begin{array}{l}\text { I } 39: \text { et qu'est-ce que tu fais lorsqu’ils te } \\
\text { disent ça? }\end{array}$ \\
\hline D $40:$ nada.. & D $40:$ rien... \\
\hline I $41:$ ¿nada? & I $41:$ rien? \\
\hline D $42:$ ¡no! & D $42:$ non! \\
\hline I $43:$ ¿no? & I $43:$ non? \\
\hline D $44:$ no no no no más porque... no & $\begin{array}{l}\text { D } 44: \text { non non non non plus parce que... on } \\
\text { ne répo[nd pas] // ou que., on ne répond } \\
\text { pas }\end{array}$ \\
\hline
\end{tabular}




\begin{tabular}{l|l}
\hline I 45: pues si... & I $45:$ bah oui... \\
\hline $\begin{array}{l}\text { D 46: pues de contestarles, de } \\
\text { decirles... pues es nada más, pues... }\end{array}$ & D $46:$ bah si on leur répond, si on leur dit... \\
(vmb) defenderse. ¿Para qué?, [es] & quoi bon ?, [c'est] chercher des problèmes, \\
estar buscando problemas, iay dios!.. & aïe dieu $! .$. [c'est] se disputer.. \\
discutiendo.. & \\
\hline
\end{tabular}

Pour des raisons de place, nous n'avons présenté ici qu'un fragment significatif de cette interaction, celui qui manifeste la rupture du veto catégorique lié au mépris sociolinguistique. Ici, le chercheur fait émerger les désignants indienne et Maria $^{12}$ par lesquels les hispanophones stigmatisent les nahuatophones (I 34). C'est ainsi que, dans la séquence suivante (D 35-D 38), les locutrices adoptent une position plus personnelle dans la remémoration du conflit. La femme la plus âgée cherche le soutien de la jeune locutrice, sa fille, afin de légitimer ses réactions affectives qui culminent avec la citation : « oui.. Pourquoi tu parles comme ça, indienne?». La stigmatisation identitaire est alors particulièrement visible, tout comme le processus cathartique qui commence par la verbalisation du conflit. I 39 cherche à connaître les réactions que les locutrices développent pour faire face à de telles tensions. Les séquences suivantes (D 40-D 46) montrent que, dans cette situation embarrassante, les locutrices répondent par un silence prolongé afin de clore l'interaction. Le silence est alors, comme on l'a dit plus haut, une stratégie d'ajustement destinée à éviter un conflit; mais il perpétue les rapports asymétriques de pouvoir et consacre la disparition du nahuatl.

Ce travail identifie les marques discursives liées à la stigmatisation d'une communauté nahua du centre du Mexique. Nous avons montré que ces réactions, tout en s'intégrant dans les interactions sociales, sont le résultat de schèmes communicatifs (Briggs, 1990). Elles jouent un rôle important dans la reconfiguration des identités collectives, en intégrant les idéologies linguistiques qui légitiment la disparition de cette langue dans la vie quotidienne des locuteurs.

Nous avons aussi montré que le discours est un outil important pour internaliser ou contester les conflits glottopolitiques ainsi que la violence symbolique de la stigmatisation identitaire. Mais il permet aussi de les comprendre. Les réactions étudiées mettent notamment en lumière les schèmes communicatifs associés au veto catégorique (Aracil, 1983). Celui-ci, considéré comme l'une des stratégies d'ajustement décrites parE. Goffman, et du fait du mutisme qui le caractérise, s'exprime à tous les niveaux du discours, mais surtout de manière pragmatique. Dans les réactions analysées, le silence se convertit

12. Le nom propre Maria est employé, au Mexique, de manière péjorative afin de désigner les femmes issues de communautés où l'on parle une langue amérindienne. Maria devient une antonomase dépréciative d'indienne. 
alors en un marqueur plein de signification et ouvre la porte à d'autres formes d'interaction. Il permet par exemple aux participants de créer diverses formes de pauses, d'espaces pour formuler des questions et thématiser le conflit.

Le positionnement empathique du chercheur favorise une catharsis identitaire pour les locuteurs. Il montre en outre que la subjectivité, dans de tels cas, est utile en termes humains mais aussi scientifiques - plus utile en tout cas que la neutralité, presque glaciale, typique d'une objectivité hyper-rationaliste (Feyerabend, 2010). La subjectivité, en d'autres termes, n'est pas ici l'antithèse de l'objectivité, mais un moyen de mieux installer cette dernière, afin de rendre compte au plus près de l'objet d'étude. Des signes de réactions discursives similaires ont été mis en évidence dans une autre communauté nahuatophone éloignée de Santa Catarina, en relation avec un sujet bien différent : les manifestations esthétiques et rituelles à San Luis Acatlan, Guerrero (Macías, 2011). L'auteur de ce dernier travail (anthropologue outsider) et moi-même nous demandons par conséquent si ces réactions ne sont pas des schèmes communicatifs liés aux secrets communautaires - dont les tabous et les rituels font partie - et que les locuteurs nahuatophones développent face aux acteurs étrangers, tout particulièrement aux chercheurs. Question qui nous invite à approfondir et à partager ces expériences dans d'autres contextes linguistiques comme dans d'autres domaines d'étude ${ }^{13}$.

\section{Références}

Alarcos Emilio, 2002, Gramática de la lengua española, Madrid, Real Academia Española, ESPASA.

ArACIL Lluís, 1983, Dir la Realitat, Barcelona, Països Catalans.

AVILÉS GonzÁlez Karla Janiré, 2011, «Aquí ya no hablan mexicano... iles da pena! Estigmas nahuas en Santa Catarina, Tepoztlán, Morelos », Entre el estigma y la resistencia. Dinámicas étnicas en tiempos de globalización, K. J. Avilés González, A. Terven éd., Mexico, CIESAS-COLMICH, p.181-216.

- 2015, "Conflits et négociations avec les locuteurs de langues en danger : un rite de passage perpétuel?», Documentation et revitalisation des "langues en danger». Épistémologie et praxis, J.-L. Léonard, K. J. Avilés González éd., Paris, Michel Houdiard.

Avilés GonzÁlez Karla Janiré, Flores Farfán José Antonio, 2010, «Défis et paradoxes de la revendication nahua à Santa Catarina, Tepoztlán, Morelos (Mexique)», Pour

13. Je tiens à remercier les évaluateurs anonymes de ce texte qui ont contribué, par leurs commentaires, à l'améliorer; l'équipe du projet IUF Morphophonologie mésoaméricaine (responsable J.-L. Léonard, CNRS - Paris 3), pour avoir pris en charge les frais d'inscription au Colloque international EASA 2012, «Uncertainty and disquiet», où une partie de ce travail a été présentée; et le programme «Investissements d'avenir » géré par l'Agence nationale de la recherche ANR-10LABX-0083 (Labex EFL), pour avoir rendu possible la rédaction de ce travail. 
une épistémologie de la sociolinguistique, H. Boyer éd., Limoges, Lambert-Lucas, p. $73-81$.

Barbet Denis, Honoré Jean-Paul, 2013, "Ce que se taire veut dire. Expressions et usages politiques du silence», Mots. Les langages du politique, $\mathrm{n}-103$, Le silence en politique, p. 7-21.

Barriga Rebeca, Martín-Butrageño Pedro éd., 2010, Historia sociolingüística de México, vol. I et II, Mexico, El Colegio de México.

BouRdieu Pierre, 2001, Langage et pouvoir symbolique, Paris, Fayard.

BOYER Henri, 2012, "L'implication du sociolinguiste "périphérique” ", Cahiers de l'Observatoire des pratiques linguistiques. Langues de France, langues en danger. Aménagement et rôle des linguistes, $\mathrm{n}^{\circ} 3$, A-L. Dotte, V. Muni Toke, J. Sibille éd., Paris, Ministère de la Culture et de la Communication, DGLFLF, p.79-85.

BRIGGS Charles, 1990, Learning how to ask. A sociolinguistic appraisal of the role of the interview in social science research, Cambridge, Cambridge University Press.

CAMACHO Fernando, 2015, "Lucha por el náhuatl de Morelos», La Jornada, en ligne [http://www.jornada.unam.mx/2015/02/21/sociedad/036n1soc] (consulté en juin 2015).

EPHRATT Michal, 2011, "Linguistic, paralinguistic and extralinguistic speech and silence », Journal of Pragmatics, ${ }^{\circ}{ }^{4}$ 33, p. 2286-2307.

Feyerabend Paul, 2010, Against Method, Londres, Verso.

FISHMAN Joshua, 1991, Reversing Language Shift, Philadelphie, Multilingual Matters.

Franco Pellotier Víctor, 2004, Oralidad y ritual matrimonial entre los amuzgos de Oaxaca, thèse en anthropologie sous la direction de Sergio Pérez Cortés, Mexico, UAM-I.

Goffman Erving, 1991, Les cadres de l'expérience, Paris, Minuit.

- 2001 [1963], Estigma. La identidad deteriorada, Buenos Aires, Amorrortu.

GUESPIN Louis, 1984, "Introduction. Matériaux pour une glottopolitique», Cahiers de linguistique sociale. Problèmes de glottopolitique, $\mathrm{n}$ 으. 7, p. 11-32.

HALE Kenneth, 1992, "On endangered languages and the safeguarding of diversity», Language, $\mathrm{n}^{\circ} 68$ (1), p. 1-3.

HILL Jane, 2001, «Language, race, and white public space », Linguistic Anthropology. A Reader, A. Duranti éd., Massachusetts, Blackwell Publishers, p. 479-492.

HILL Jane, HILL Keneth, 1999, Hablando mexicano, Mexico, CIESAS-INI.

Hymes Dell, 1974, "Hacia etnografías de la comunicación », Antología de estudios de etnolingüística y sociolingüística, P. Garvin, Y. Lastra éd., Mexique, UNAM, IIA.

IRVING Judith, 2001, "Formality and informality in communicative events", Linguistic Anthropology. A Reader, A. Duranti éd., Massachusetts, Blackwell Publishers, p. $172-187$.

LÉonARd Jean-Léo, AvilÉs González Karla Janiré éd., 2015, Documentation et revitalisation des «langues en danger». Épistémologie et praxis, Paris, Michel Houdiard.

MACíAS Eugenia, 2011, «Resistencia indígena y experiencia estética. Los mandiles de las fiestas de mayo en Acatlán-Chilapa, Guerrero », Entre el estigma y la resistencia. Dinámicas étnicas en tiempos de globalización, K. J. Avilés González, A. Terven éd., Mexico, CIESAS, p. 71-119. 
MuÑoz Héctor, 1987, «Testimonios metalingüísticos de un conflicto intercultural. ¿Reivindicación o sólo representación de la cultural otomí?», Funciones sociales y conciencia del lenguaje, H. Muñoz éd., Jalapa (Mexique), Universidad Veracruzana. NinYoles Rafael, 1997 [1971], Idioma y prejudici, Barcelone, Quaderns Tres i Quatre.

Reguillo Rossana, 2000, "La clandestina centralidad de la vida cotidiana », La vida cotidiana y su espacio-temporalidad, A. Lindón éd., Barcelone, Anthropos.

SiERRA Teresa, 2012, "Redefiniendo los espacios de género desde la diversidad cultural. Las mujeres indígenas frente a la justicia y los derechos en México y América Latina», Espacios de Género. ADLAF Congreso Anual 2012, J. Strëbele-Gregor, D. Wollrad éd., Buenos Aires, Foro Nueva Sociedad.

Silverstein Michael, 1996, "Monoglot "standard" in America. Standardization and metaphors of linguistic hegemony », The Matrix of Language. Contemporary Linguistic Anthropology, D. Brenneis, K. Macaulay éd., Colorado, Westview Press.

Skutnabb-Kangas Tove, Luisa Maffi y David Harmon, 2003, Sharing a World of Difference. The Earth's Linguistic, Cultural and Biological Diversity, Paris, UNESCO. 